\title{
An Original and Additional Mathematical Model Characterizing a Bayesian Approach to Decision Theory
}

\author{
Angelini Pierpaolo \\ Correspondence: Angelini Pierpaolo, Dipartimento Di Scienze Statistiche, Universita' La Sapienza Di Roma, Italy. E- \\ mail: pier.angelini@uniroma1.it
}

Received: March 13, 2019 Accepted: April 8, 2019 Online Published: April 10, 2019

doi:10.5539/jmr.v11n3p1 URL: https://doi.org/10.5539/jmr.v11n3p1

\begin{abstract}
We propose an original mathematical model according to a Bayesian approach explaining uncertainty from a point of view connected with vector spaces. A parameter space can be represented by means of random quantities by accepting the principles of the theory of concordance into the domain of subjective probability. We observe that metric properties of the notion of $\alpha$-product mathematically fulfill the ones of a coherent prevision of a bivariate random quantity. We introduce fundamental metric expressions connected with transformed random quantities representing changes of origin. We obtain a posterior probability law by applying the Bayes' theorem into a geometric context connected with a two-dimensional parameter space.
\end{abstract}

Keywords: vector space, parameter space, antisymmetric tensor, $\alpha$-product, $\alpha$-norm, change of origin

\section{Introduction}

We make clear some essential points in order to found our geometric model. A quantity is random for a given individual at a certain instant when he does not know its true value. Therefore, he is in doubt between two or more than two possible values. One and only one of these possible values will be true "a posteriori" (Pompilj, 1957). Each possible value of a random quantity is an event. Thus, every finite partition of incompatible and exhaustive events represents a random quantity. It follows that every event is a particular random quantity because it admits only two possible values, 1 and 0 . We show a model where random quantities are studied by using the logic of certainty and the logic of the probable. The logic of certainty does not use the notion of probability (Jeffreys, 1961), (de Finetti, 1982a). The logic of certainty is characterized by two different and extreme aspects. The first aspect deals with situations of non-knowledge or ignorance or uncertainty. These situations determine a set of possible alternatives of a random quantity with respect to a given set of information. The second aspect deals with definitive certainty expressed in the form of what is certainly true or certainly false. Thus, every possible numerical value of a random quantity definitively becomes 0 or 1 . Conversely, the notion of probability is of interest to an intermediate aspect which is included between the two extreme aspects characterizing the logic of certainty (Good, 1962), (Koopman, 1940). Indeed, probability is distributed as a mass by a given individual over the domain of possible alternatives before knowing which is the true alternative to be verified "a posteriori". Probability is an extralogical notion in the sense that it is outside of the logic of certainty (Ramsey, 1960), (Savage, 1954). Probability must subjectively be distributed in a coherent way. Conditions of coherence are objective (de Finetti, 2011).

\section{The Point Under Discussion}

When we deal with a decision-maker choosing an action under conditions of uncertainty we use a mathematical model coinciding with a quadruple of elements given by

$$
(\Omega, A, L(\theta, a), K),
$$

where $\Omega$ is a parameter space, $A$ is a space of possible actions, $L(\theta, a)$ is a loss function, $K$ is a functional which must be minimized. It is essential to note that we deal with a mathematical model characterizing a Bayesian approach when we introduce a probability law $\mathbf{P}$ with respect to $\Omega$, so we consider a probability space given by

$$
\left(\Omega, \mathcal{A}_{\Omega}, \mathbf{P}\right)
$$

instead of $\Omega$, where $\mathcal{A}_{\Omega}$ is a $\sigma$-algebra on $\Omega$. In general, possible elements of $\Omega$ are known while it is not known which is the true parameter of $\Omega$ among all possible parameters. It is denoted by $\theta^{*} \in \Omega$ and it represents the real explanation of the phenomenon under consideration. A generic element of $\Omega$ is denoted by $\theta$. When a decision-maker chooses an action $a \in A$ we observe an outcome denoted by $L(\theta, a)$ depending on two elements, $\theta \in \Omega$ and $a \in A$. Thus, we obtain a space of possible outcomes when $\theta \in \Omega$ and $a \in A$ vary into $\Omega$ and $A$. Such outcomes are uncertain and they must 
be compared because any decision-maker always tries to get the best possible outcome (Barlow, Claroti \& Spizzichino, 1993). In general, it turns out to be

$$
L(\theta, a)>0, \text { if } \theta \neq a,
$$

while we have

$$
L(\theta, a)=0, \text { if } \theta=a .
$$

Thus, an optimal choice is theoretically given by

$$
K(L(\theta, a))=\text { minimum for } a \in A .
$$

On the other hand, a given phenomenon can be studied by means of a statistical experiment whose mathematical representation is expressed by

$$
e=\left(\mathcal{Z}, \mathbf{P}_{\theta}, \theta \in \Omega\right),
$$

where $\mathcal{Z}$ is a set of possible experimental outcomes, $\mathbf{P}_{\theta}$ is a probability law with respect to $\left(\mathcal{Z}, \mathcal{A}_{\mathcal{Z}}\right)$ and $\theta$ is a parameter of $\Omega$. We have that $\mathcal{A}_{\mathcal{Z}}$ is a $\sigma$-algebra on $\mathcal{Z}$. If a statistical experiment is carried out, then it is denoted by $z \in \mathcal{Z}$. A mathematical model of a statistical decision is obtained by considering (1) and (6) in a jointly fashion, so it turns out to be

$$
(\Omega, A, L(\theta, a), K), \quad e=\left(\mathcal{Z}, \mathbf{P}_{\theta}, \theta \in \Omega\right),
$$

where the set $\Omega$ characterizing $e$ is the same of the one characterizing (1). Problems of statistical inference are problems that can be represented by means of mathematical models of statistical decisions (Forcina, 1982). In particular, we are interested in structural or hypothetical problems according to which it is essential to get information about $\theta^{*} \in \Omega$ by using a conditional probability law of $\Theta$ given that an experimental outcome $z \in \mathcal{Z}$ has occurred. Therefore, if $(\Theta, Z)$ is a continuous random quantity, $\pi(\cdot)$ is a probability density function of $\Theta$ and $p_{\theta}(\cdot)$ is a probability density function of $Z$ corresponding to $\mathbf{P}_{\theta}$ for each $\theta \in \Omega$, then we have

$$
\pi(\theta ; z)=\frac{\pi(\theta) p_{\theta}(z)}{\int_{\Omega} \pi(\theta) p_{\theta}(z) d \theta}
$$

according to the Bayes' theorem, where $\pi(\theta ; z)$ is a conditional probability density function of $\Theta$ given that $Z=z$ has occurred. We note that $p_{\theta}(z)$ is a likelihood function (Basu, 1975). An experimental outcome $z \in \mathcal{Z}$ changes an "a priori” probability law given by $\pi(\theta)$ into an "a posteriori” probability law given by $\pi(\theta ; z)$. This "a posteriori” probability law is meaningful but we are also interested in one or more than one parameter, so we have to summarize it. By leaving problems of statistical inference in order to consider more general problems of statistical decisions according to a Bayesian approach we note that a decision-maker chooses an action $a \in A$ when a statistical experiment $z \in \mathcal{Z}$ is carried out. Thus, our variable is $a \in A$ while $z \in \mathcal{Z}$ is known. If an optimal decision exists, then it is given by

$$
K(L(\cdot, a))=\text { minimum for } a \in A .
$$

If $K$ coincides with the criterion of the expected value, then a decision-maker has to minimize a final expected loss. Thus, given

$$
\rho(a ; z)=\mathbb{E}(L(\Theta, a) \mid Z=z)=\int_{\Omega} L(\theta, a) \pi(\theta ; z) d \theta,
$$

it turns out to be

$$
\rho(a ; z)=\text { minimum for } a \in A .
$$

Having said that, it is possible to show a geometric and original Bayesian approach connected with decision theory under conditions of uncertainty. We propose an additional mathematical model explaining uncertainty from another point of view (deGroot, 1962), (Piccinato, 1986). On the other hand, every Bayesian criterion properly claims a possibility of formalizing uncertainty in all its aspects. Nevertheless, it is not certainly appropriate to incorporate within uncertainty all its components in an indistinct fashion. It is then necessary to separate an analysis depending on experimental information from an analysis that does not depend on experimental information. When we say that one and only one point of $\Omega$ represents the real explanation of the phenomenon under consideration, we mean that every point of $\Omega$ is a single random event that could occur or not. Every single random event belongs to a given random quantity. Thus, it is possible to know two different ways in order to deal with uncertainty of a random event. Firstly, every random event can be viewed as a single random element, so we can directly assign to it a subjective probability. This means that a random event is not a measurable set characterized by a conventional notion of a mathematical nature. Secondly, every random event can be interpreted as a basic random element contained in a greater random element coinciding with a random variable 
(measurable function). We are interested in knowing only this greater random element. In particular, we are interested in knowing which is the probability distribution of this greater random element into a probability space characterized by a $\sigma$-algebra. We consequently believe that a complete Bayesian approach to decision theory must not only be based on a probability distribution with respect to $\Omega$. It must also consider $\Omega$ from a geometric point of view. Indeed, it is possible to get useful information about points of $\Omega$ by considering both experimental outcomes and subjective degrees of belief. On the other hand, it is not in general possible to deduce with certainty the true value of $\theta^{*} \in \Omega$ from an experimental outcome. These two different ways characterizing uncertainty are evidently complementary. The former is more satisfactory from a conceptual point of view connected with the notion of subjective probability. Anyway, $\Omega$ is provided with a geometric structure introduced after taking under consideration all possible alternatives of a random quantity representing it. All alternatives are logically possible for a given individual at a certain instant with a given set of information.

\section{Vector Representation of an One-Dimensional Parameter Space}

An one-dimensional parameter space $\Omega \subseteq \mathbb{R}$ can be represented by means of a univariate random quantity. We can consider different univariate random quantities that are elements of a set of univariate random quantities denoted by ${ }_{(1)} S$. We denote by $\Omega \in{ }_{(1)} S$ one of these univariate random quantities. Every random quantity belonging to the set ${ }_{(1)} S$ is represented by a vector belonging to $E_{m}$, where $E_{m}$ is an $m$-dimensional vector space over the field $\mathbb{R}$ of real numbers. An orthonormal basis of $E_{m}$ is denoted by $\left\{\mathbf{e}_{j}\right\}, j=1, \ldots, m$. The different possible values of every random quantity of ${ }_{(1)} S$ are $m$ in number. It turns out to be ${ }_{(1)} S \subset E_{m}$. A univariate quantity $\Omega$ is really random for a given decision-maker when he is in doubt between two or more than two possible values of $\Omega$ belonging to the set $\mathcal{I}(\Omega)=\left\{\theta^{1}, \ldots, \theta^{m}\right\}$. Each possible value of $\Omega$ is an event. Only one of them will occur "a posteriori". It is not at all a logical restriction to consider a random quantity as a finite partition of incompatible and exhaustive events. Hence, it must be possible to consider every single event of a finite partition of events as a statement such that, by betting on it, we can establish whether the bet has been won or lost (de Finetti, 1981). Nevertheless, if we wonder which is the event that will occur among an infinite number of them we can never verify if each statement representing a single event is true or false. These statements are infinite in number. Therefore, they are conceptually meaningless. Thus, the same symbol $\mathbf{P}$ is used in order to denote both a prevision of a random quantity and a probability of an event (de Finetti, 1982b). It is essential to note that each $\theta^{i}, i=1, \ldots, m$, can also represent a cell midpoint when $\Omega$ is a bounded (from above and below) continuous parameter space. On the other hand, it is possible to dichotomize a bounded (from above and below) continuous random quantity by giving origin to different dichotomic random quantities. The different possible values of $\Omega$ belonging to the set $\mathcal{I}(\Omega)$ coincide with the different components of a vector $\omega \in E_{m}$ and they can indifferently be denoted by a covariant or contravariant notation after choosing an orthonormal basis of $E_{m}$. We should exactly speak of components of $\omega$ having upper or lower indices because we deal with an orthonormal basis of $E_{m}$. Indeed, to use the terms covariant and contravariant is geometrically meaningless because the covariant components of $\omega$ coincide with the contravariant ones. Nevertheless, it is appropriate to use this notation because a particular meaning connected with these components will be introduced. Having said that, we will continue to use these terms. Thus, we choose a contravariant notation with respect to the components of $\omega$ so it is possible to write $\omega=\left(\theta^{i}\right)$. We choose a covariant notation with respect to the components of $\mathbf{p}$ so it is possible to write $\mathbf{p}=\left(p_{i}\right)$, where $p_{i}$ represents a subjective probability assigned to $\theta^{i}, i=1, \ldots, m$, by a given decision-maker. Hence, different decision-makers whose state of knowledge is hypothetically identical may choose different $p_{i}$. Indeed, each of them may subjectively give greater attention to certain circumstances than to others (Kyburg jr. \& Smokler, 1964). A given decision-maker is into the domain of the logic of certainty when he considers only $\omega \in E_{m}$, while he is into the domain of the logic of the probable when he considers an ordered pair of vectors given by $(\omega, \mathbf{p})$. Thus, a prevision of $\Omega$ is given by

$$
\mathbf{P}(\Omega)=\bar{\Omega}=\theta^{i} p_{i},
$$

where we imply the Einstein summation convention. This prevision is coherent when we have $0 \leq p_{i} \leq 1, i=1, \ldots, m$, as well as $\sum_{i=1}^{m} p_{i}=1$ (Coletti \& Scozzafava, 2002). This implies that a coherent prevision of $\Omega$ always satisfies the inequality inf $\mathcal{I}(\Omega) \leq \mathbf{P}(\Omega) \leq \sup \mathcal{I}(\Omega)$ and it is also linear. A coherent prevision of $\Omega$ can be expressed by means of the vector $\bar{\omega}=\left(\bar{\omega}^{i}\right)$ that allows us of defining a transformed random quantity denoted by ${ }_{\Omega} t$ : it is represented by the vector ${ }_{\omega} \mathbf{t}=\omega-\bar{\omega}$ whose contravariant components are given by

$$
\omega^{i} t^{i}=\theta^{i}-\bar{\omega}^{i}
$$

This linear transformation of $\Omega$ is a change of origin. A coherent prevision of the transformed random quantity ${ }_{\Omega} t$ is given by

$$
\mathbf{P}\left({ }_{\Omega} t\right)=\left(\theta^{i}-\bar{\omega}^{i}\right) p_{i}=0
$$

The $\alpha$-norm of the vector $\omega$ is expressed by

$$
\|\omega\|_{\alpha}^{2}=\left(\theta^{i}\right)^{2} p_{i} .
$$


It is the square of the quadratic mean of $\Omega$. It turns out to be $\|\omega\|_{\alpha}^{2} \geq 0$. In particular, when the possible values of $\Omega$ are all null one writes $\|\omega\|_{\alpha}^{2}=0$ : this is a degenerate case that we exclude. Hence, it is possible to say that the $\alpha$-norm of the vector $\omega$ is strictly positive. The $\alpha$-norm of the vector representing $\Omega_{\Omega} t$ is given by

$$
\left\|_{\omega} \mathbf{t}\right\|_{\alpha}^{2}=\left({ }_{\omega} t^{i}\right)^{2} p_{i}=\sigma_{\Omega}^{2} .
$$

It represents the variance of $\Omega$ in a vectorial fashion (Castellano, 1989). We will later explain why we use the term $\alpha$-norm.

\section{Invariance Under Translation of a Transformed Random Quantity}

It is possible to prove a very important property connected with a vector representing a univariate transformed random quantity. This property is intrinsic because it does not depend on the choice of a particular orthonormal basis of $E_{m}$. Thus, it is at first possible to introduce the vector

$$
\omega^{\prime}=\omega-g(\omega),
$$

where $g$ is a linear transformation from $E_{m}$ to $E_{m}$ whose associated matrix is non-singular. Given a generic vector $\tau \in E_{m}$, with respect to the vector $\omega$, the translation $\mathcal{T}_{\tau}$ is expressed by

$$
\mathcal{T}_{\tau}(\omega): \omega \Rightarrow \omega+\tau .
$$

Given $\tau \in E_{m}$, with respect to the vector $\omega^{\prime}$, the translation $\mathcal{T}_{\tau}$ is expressed by

$$
\mathcal{T}_{\tau}\left(\omega^{\prime}\right): \omega^{\prime} \Rightarrow \omega^{\prime}+\tau .
$$

Given (17), with respect to the vector $\omega^{\prime}$, the translation $\mathcal{T}_{\tau}$ can be expressed by

$$
\mathcal{T}_{\tau}\left(\omega^{\prime}\right)=\mathcal{T}_{\tau}[\omega-g(\omega)]=\mathcal{T}_{\tau}(\omega)-\mathcal{T}_{\tau}[g(\omega)] .
$$

Since $g$ is a linear transformation, it is possible to write

$$
\mathcal{T}_{\tau}\left(\omega^{\prime}\right)=\mathcal{T}_{\tau}(\omega)-g\left[\mathcal{T}_{\tau}(\omega)\right] .
$$

The translation that appears as a minuend into (21) is given by (18). When one defines the translation that appears as a subtrahend into (21) one writes

$$
g\left[\mathcal{T}_{\tau}(\omega)\right]: g(\omega) \Rightarrow g(\omega+\tau) .
$$

After considering (17), (18) and (22) into (19), where (19) is made clear by means of (21), it is possible to obtain

$$
\mathcal{T}_{\tau}\left(\omega^{\prime}\right): \omega-g(\omega) \Rightarrow \omega+\tau-g(\omega+\tau) .
$$

It is necessary to establish which is the condition according to which $\omega^{\prime}=\omega-g(\omega)$ is invariant under translation. It is expressed by

$$
\omega-g(\omega)=\omega+\tau-g(\omega+\tau)
$$

From linearity of $g$ it follows that it is possible to write

$$
\omega-g(\omega)=\omega+\tau-g(\omega)-g(\tau) .
$$

Therefore, when it turns out to be

$$
\tau=g(\tau)
$$

it is possible to say that $\omega^{\prime}$ is invariant under translation. The equality expressed by (26) exists when $g$ is a linear operator viewed as an identity transformation applied to $E_{m}$. It exists when $\tau$ is a zero vector. Nevertheless, they are two degenerate cases. It exactly exists when $\tau$ is a constant, that is to say, when $\tau$ represents a coherent prevision of $\Omega$ in a vectorial fashion. Hence, all contravariant components of $\tau$ are equal. From the vector $\omega^{\prime}=\omega-g(\omega)$ it follows the vector

$$
{ }_{\omega} \mathbf{t}^{\prime}={ }_{\omega} \mathbf{t}-g\left({ }_{\omega} \mathbf{t}\right) .
$$

It represents a univariate transformed random quantity. Given ${ }_{\omega} \mathbf{t}=\omega-\bar{\omega}$, it is possible to write

$$
{ }_{\omega} \mathbf{t}^{\prime}=(\omega-\bar{\omega})-g(\omega-\bar{\omega})=\omega-\bar{\omega}-g(\omega)+g(\bar{\omega}) .
$$

Since we have $g(\bar{\omega})=\bar{\omega}$, it turns out to be

$$
{ }_{\omega} \mathbf{t}^{\prime}=\omega-g(\omega),
$$


that is to say, the vector representing a univariate transformed random quantity coincides with the vector $\omega^{\prime}$. It is possible to prove that ${ }_{\omega} \mathbf{t}^{\prime}$ is invariant under translation. With regard to ${ }_{\omega} \mathbf{t}$ we have

$$
\mathcal{T}_{\epsilon}\left({ }_{\omega} \mathbf{t}\right):{ }_{\omega} \mathbf{t} \Rightarrow{ }_{\omega} \mathbf{t}+\epsilon=\omega-\bar{\omega}+\epsilon,
$$

where $\epsilon \in E_{m}$ is a generic vector. With regard to ${ }_{\omega} \mathbf{t}^{\prime}$ we have

$$
\mathcal{T}_{\epsilon}\left({ }_{\omega} \mathbf{t}^{\prime}\right):{ }_{\omega} \mathbf{t}^{\prime} \Rightarrow{ }_{\omega} \mathbf{t}^{\prime}+\epsilon=\omega-g(\omega)+\epsilon,
$$

with $\epsilon \in E_{m}$. By writing

$$
\omega-\bar{\omega}+\epsilon=\omega-g(\omega)+\epsilon
$$

one obtains $g(\omega)=\bar{\omega}=\left(\bar{\omega}^{i}\right)$. Therefore, the vector representing a univariate transformed random quantity is invariant under translation. It is finally possible to write

$$
\mathcal{T}_{\epsilon}\left({ }_{\omega} \mathbf{t}\right)=\mathcal{T}_{\epsilon}\left({ }_{\omega} \mathbf{t}^{\prime}\right)={ }_{\omega} \mathbf{t} .
$$

The true parameter of a parameter space representing the real explanation of the phenomenon under consideration is always the same. Therefore, it does not depend on a particular parameterization or formulation of the problem under consideration. Different parameterizations are equivalent, so a given decision-maker can choose one of them. The true parameter is consequently invariant.

\section{Tensor Representation of a Two-Dimensional Parameter Space}

A two-dimensional parameter space $\Omega \subseteq \mathbb{R}^{2}$ can be represented by means of a bivariate random quantity. We denote by ${ }_{(2)} S^{(2)}$ a set of bivariate random quantities and by $\Omega_{12} \equiv\left\{{ }_{1} \Omega,{ }_{2} \Omega\right\}$ a generic bivariate random quantity of this set. A pair of univariate random quantities $\left({ }_{1} \Omega,{ }_{2} \Omega\right)$ evidently represents an ordered pair of univariate random quantities that are the components of $\Omega_{12}$. Each element of ${ }_{(2)} S^{(2)}$ can be represented by an affine tensor of order 2 denoted by $T \in{ }_{(2)} S^{(2)}$. Moreover, it turns out to be ${ }_{(2)} S^{(2)} \subset E_{m}^{(2)}$, where we have $E_{m}^{(2)}=E_{m} \otimes E_{m}$. An orthonormal basis of $E_{m}$ is denoted by $\left\{\mathbf{e}_{j}\right\}$, $j=1, \ldots, m$. Therefore, the possible values of $\Omega_{12}$ coincide with the numerical values of the components of $T$. A vector space denoted by $E_{m}$ has a dimension equal to $m$. The number of the different possible values of every univariate random quantity of $\Omega_{12}$ is equal to $m$. Thus, $T$ is an element of an $m^{2}$-dimensional vector space. We can represent the possible values of $\Omega_{12}$ by means of an orthonormal basis of $E_{m}$. These values coincide with the contravariant components of $T$ so it is possible to write

$$
T={ }_{(1)} \omega \otimes{ }_{(2)} \omega={ }_{(1)} \theta_{(2)}^{i_{1}} \theta^{i_{2}} \mathbf{e}_{i_{1}} \otimes \mathbf{e}_{i_{2}} .
$$

The tensor representation of $\Omega_{12}$ expressed by (34) depends on $\left({ }_{1} \Omega,{ }_{2} \Omega\right.$ ). Indeed, if one considers a different ordered pair $\left.{ }_{2} \Omega,{ }_{1} \Omega\right)$ of univariate random quantities one obtains a different tensor representation of $\Omega_{12}$. It is expressed by

$$
T={ }_{(2)} \omega \otimes{ }_{(1)} \omega={ }_{(2)} \theta_{(1)}^{i_{2}} \theta^{i_{1}} \mathbf{e}_{i_{2}} \otimes \mathbf{e}_{i_{1}}
$$

because the tensor product is not commutative (McCullagh, 1987). Therefore, the components of $T$ expressed by (35) are not the same of the ones expressed by (34). Both these formulas express an affine tensor of order 2 whose components are different. In particular, we could consider two vectors of $E_{3}$

$$
{ }_{(1)} \omega={ }_{(1)} \theta^{1} \mathbf{e}_{1}+{ }_{(1)} \theta^{2} \mathbf{e}_{2}+{ }_{(1)} \theta^{3} \mathbf{e}_{3}
$$

and

$$
{ }_{(2)} \omega={ }_{(2)} \theta^{1} \mathbf{e}_{1}+{ }_{(2)} \theta^{2} \mathbf{e}_{2}+{ }_{(2)} \theta^{3} \mathbf{e}_{3}
$$

in order to realize that it turns out to be ${ }_{(1)} \omega \otimes{ }_{(2)} \omega \neq{ }_{(2)} \omega \otimes{ }_{(1)} \omega$ by summing over all values of the indices. Then, we must consider (34) and (35) in a jointly fashion in order to release a tensor representation of $\Omega_{12}$ from any ordered pair of univariate random quantities that can be considered, $\left({ }_{1} \Omega,{ }_{2} \Omega\right)$ or $\left({ }_{2} \Omega,{ }_{1} \Omega\right)$. In fact, when $m=3$ and we express $T$ by means of (34) and (35) we observe that three of nine summands are equal. It is consequently possible to say that the possible values of a bivariate random quantity must be expressed by the components of an antisymmetric tensor of order 2. It is expressed by

$$
T=\sum_{i_{1}<i_{2}}\left({ }_{(1)} \theta^{i_{1}}{ }_{(2)} \theta^{i_{2}}-{ }_{(1)} \theta^{i_{2}}{ }_{(2)} \theta^{i_{1}}\right) \mathbf{e}_{i_{1}} \otimes \mathbf{e}_{i_{2}} .
$$

The number of the components of an antisymmetric tensor of order 2 is evidently different from the one of the components of an affine tensor of the same order. Thus, a tensor representation based on an antisymmetric tensor of order 2 does not depend either on $\left({ }_{1} \Omega,{ }_{2} \Omega\right)$ or $\left({ }_{2} \Omega, \Omega\right)$. We choose it in order to represent a generic bivariate random quantity $\Omega_{12}$ in a 
geometrical fashion. Therefore, ${ }_{12} f$ is an antisymmetric tensor of order 2 called the tensor of possible values of $\Omega_{12}$. The contravariant components of ${ }_{12} f$ expressed by

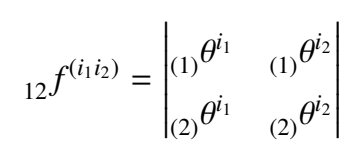

represent the possible values of $\Omega_{12}$ in a tensorial fashion. When these components have equal indices it follows that they are equal to 0 . It is evident that a vector space of the antisymmetric tensors of order 2 is not $m^{2}$-dimensional but it is $\left(\begin{array}{c}m \\ 2\end{array}\right)$-dimensional. Now, we must introduce probability into this geometric representation of $\Omega_{12}$. This means that a given decision-maker must distribute a mass over the possible alternatives coinciding with the possible values of $\Omega_{12}$. Therefore, he leaves the domain of the logic of certainty in order to go into the domain of the logic of the probable. We say that the tensor of the joint probabilities $p=\left(p_{i_{1} i_{2}}\right)$ is an affine tensor of order 2 whose covariant components represent those probabilities connected with ordered pairs of components of vectors representing the marginal univariate random quantities ${ }_{1}$ and ${ }_{2} \Omega$ of $\Omega_{12}$. Then, a coherent prevision of $\Omega_{12}$ is expressed by

$$
\mathbf{P}\left(\Omega_{12}\right)=\bar{\Omega}_{12}={ }_{(1)} \theta_{(2)}^{i_{1}} \theta^{i_{2}} p_{i_{1} i_{2}},
$$

so it is also possible to consider an affine tensor of order 2 denoted by ${ }_{12} \bar{\omega}$ whose contravariant components are expressed by ${ }_{12} \bar{\theta}^{i} i_{2}$. They are all equal. In order to define the covariant components of ${ }_{12} f$ we must consider those vector homographies that allow us of passing from the contravariant components of a type of vector to the covariant components of another type of vector by means of the tensor of the joint probabilities under consideration. Indeed, the covariant components of ${ }_{12} f$ represent those probabilities connected with the possible values of each marginal univariate random quantity of $\Omega_{12}$. These components are obtained by summing the probabilities connected with the ordered pairs of components of ${ }_{(1)} \omega$ and ${ }_{(2)} \omega$ : putting the joint probabilities into a two-way table we consider the totals of each row and the totals of each column of the table as covariant components of ${ }_{12} f$. In analytic terms we have ${ }_{(1)} \theta^{i_{1}} p_{i_{1} i_{2}}={ }_{(1)} \theta_{i_{2}}$ and ${ }_{(2)} \theta^{i_{2}} p_{i_{1} i_{2}}={ }_{(2)} \theta_{i_{1}}$ by virtue of a particular convention that we introduce: when the covariant indices to right-hand side vary over all their possible values we obtain two sequences of values representing those probabilities connected with the possible values of each marginal univariate random quantity of $\Omega_{12}$. They are the covariant components of ${ }_{12} f$. It turns out to be

$$
{ }_{12} f_{\left(i_{1} i_{2}\right)}=\left|\begin{array}{ll}
{ }_{(1)} \theta_{i_{1}} & { }_{(1)} \theta_{i_{2}} \\
{ }_{(2)} \theta_{i_{1}} & { }_{(2)} \theta_{i_{2}}
\end{array}\right|=\left|\begin{array}{ll}
{ }_{(1)} \theta^{i_{2}} p_{i_{2} i_{1}} & { }_{(1)} \theta^{i_{1}} p_{i_{1} i_{2}} \\
{ }_{(2)} \theta^{i_{2}} p_{i_{2} i_{1}} & { }_{(2)} \theta^{i_{1}} p_{i_{1} i_{2}}
\end{array}\right| .
$$

The covariant indices of the tensor $p$ can be interchanged when it is necessary so we have, for instance, ${ }_{(1)} \theta^{i_{1}} p_{i_{1} i_{2}}=$ ${ }_{(1)} \theta^{i_{1}} p_{i_{2} i_{1}}$. Each ordered pair of vectors $\left(_{(1)} \omega,{ }_{(2)} \omega\right)$ mathematically determines an affine tensor of order 2 when a given decision-maker is into the subjective domain of the logic of the probable. Each ordered pair of vectors ${ }_{(1)} \omega$, $\left.{ }_{(2)} \omega\right)$ represents two univariate random quantities, $\Omega$ and ${ }_{2} \Omega$, into $E_{m}$ (Pistone, Riccomagno \& Wynn, 2001). Both these univariate random quantities belong to the set denoted by ${ }_{(2)} S^{(1)}$ so it turns out to be ${ }_{(2)} S^{(1)} \subset E_{m}$. On the other hand, it is possible to write ${ }_{(2)} S^{(1)} \otimes_{(2)} S^{(1)}={ }_{(2)} S^{(2)}$, so we reach a vector space of the antisymmetric tensors of order 2 by anti-symmetrization. It is denoted by ${ }_{(2)} S^{(2) \wedge}$. We have evidently ${ }_{(2)} S^{(2) \wedge} \subset E_{m}^{(2) \wedge}$. We will show that a metric defined on ${ }_{(2)} S^{(2) \wedge}$ is a consequence of a metric defined on ${ }_{(2)} S^{(1)}$. When we observe that the number of the components of an antisymmetric tensor of order 2 decreases by passing from an affine tensor of order 2 to an antisymmetric tensor of the same order we say that this thing is useful in order to satisfy simplification and compression needs. Nevertheless, it is essential to note a very important point: this does not mean that the original structure of the random quantity under consideration changes. It remains unchanged. Only the number of elements that we consider by means of a tensorial representation decreases. The original elements of the random quantity under consideration do not disappear. Indeed, we will show that they are fully considered in order to establish quantitative relationships between random quantities. Therefore, it is possible to compress elements of a random quantity without changing conceptual terms of the problem under consideration.

\section{A Metric on an One-Dimensional Parameter Space}

A set of univariate random quantities that are the components of bivariate random quantities is denoted by ${ }_{(2)} S^{(1)} \subset E_{m}$. It is a vector space smaller than $E_{m}$ because each $m$-tuple of real numbers is always a sequence of $m$ different numbers. Thus, even when a given $m$-tuple is a result of the addition of two $m$-tuples we must obtain a sequence of $m$ different numbers because ${ }_{(2)} S^{(1)}$ is closed under addition of two elements of it. If this thing does not happen, then a random quantity evidently changes its structure. Univariate random quantities are represented by two vectors, ${ }_{(1)} \omega$ and ${ }_{(2)} \omega$, belonging to $E_{m}$. A given decision-maker deals with two ordered $m$-tuples when he is into the objective domain of the logic of certainty. 
An affine tensor $p$ of order 2 must be added to the two vectors under consideration when it is necessary to pass from the domain of the logic of certainty to the one of the logic of the probable. Therefore, it is always necessary to consider a triple of elements. We transform ${ }_{(2)} \omega$ into ${ }_{(2)} \omega^{\prime}$ by means of the tensor $p$. Hence, it is possible to write the following dot product

$$
{ }_{(1)} \omega \cdot{ }_{(2)} \omega^{\prime}={ }_{(1)} \theta^{i_{1}}{ }_{(2)} \theta^{i_{2}} p_{i_{1} i_{2}}={ }_{(1)} \theta_{(2)}^{i_{1}} \theta_{i_{1}}
$$

We note that

$$
{ }_{(2)} \theta_{i_{1}}={ }_{(2)} \theta^{i_{2}} p_{i_{1} i_{2}}={ }_{(2)} \omega^{\prime}
$$

is a vector homography whose expressions are obtained by applying the Einstein summation convention. Then, the $\alpha$ product of two vectors, ${ }_{(1)} \omega$ and ${ }_{(2)} \omega$, is defined as a dot product of two vectors, ${ }_{(1)} \omega$ and ${ }_{(2)} \omega^{\prime}$, so we write

$$
{ }_{(1)} \omega \odot_{(2)} \omega={ }_{(1)} \omega \cdot{ }_{(2)} \omega^{\prime} \text {. }
$$

In particular, the $\alpha$-norm of the vector ${ }_{(1)} \omega$ is given by

$$
\left\|_{(1)} \omega\right\|_{\alpha}^{2}={ }_{(1)} \theta^{i_{1}}{ }_{(1)} \theta^{i_{1}} p_{i_{1} i_{1}}={ }_{(1)} \theta_{(1)}^{i_{1}} \theta_{i_{1}} .
$$

Now, we can explain why we use this term: we use it because we refer to the $\alpha$-criterion of concordance introduced by Gini (Gini, 1926, 1966). There actually exist different criteria of concordance shown by Gini in addition to the $\alpha$ criterion. Nevertheless, by considering quadratic measures of concordance it always suffices to use the $\alpha$-criterion (Gili $\&$ Bettuzzi, 1986). When we pass from the notion of $\alpha$-product to the one of $\alpha$-norm we say that the corresponding possible values of the two univariate random quantities under consideration are equal. Moreover, we say that the corresponding probabilities are equal. Therefore, the covariant components of the tensor $p=\left(p_{i_{1} i_{2}}\right)$ having different numerical values as indices are null. Thus, we say that the absolute maximum of concordance is realized. Hence, it is evidently possible to elaborate a geometric, original and extensive theory of multiple random quantities by accepting the principles of the theory of concordance into the domain of subjective probability. This acceptance is well-founded because the definition of concordance is implicit as well as the one of prevision of a random quantity, and in particular, of probability of an event. Indeed, these definitions are based on criteria which permit to measure them. Given the vector $\epsilon={ }_{(1)} \omega+b_{(2)} \omega$, its $\alpha$-norm is expressed by

$$
\left.\|\epsilon\|_{\alpha}^{2}=\left\|_{(1)} \omega\right\|_{\alpha}^{2}+2 b{\left({ }_{(1)}\right.}^{2} \odot_{(2)} \omega\right)+b^{2}\left\|_{(2)} \omega\right\|_{\alpha}^{2} .
$$

It is always possible to write $\|\epsilon\|_{\alpha}^{2} \geq 0$. Moreover, the right-hand side of (44) is a quadratic trinomial whose variable is $b \in \mathbb{R}$, so we must consider a quadratic inequation. All real numbers fulfill the condition stated in the form $\|\epsilon\|_{\alpha}^{2} \geq 0$. This means that the discriminant of the associated quadratic equation is non-positive. We write

$$
\Delta_{b}=4\left[\left(_{(1)} \omega \odot_{(2)} \omega\right)^{2}-\|\|_{(1)} \omega\left\|_{\alpha}^{2}\right\|_{(2)} \omega \|_{\alpha}^{2}\right] .
$$

Given $\Delta_{b} \leq 0$, it turns out to be

$$
\left.{ }_{(1)} \omega \odot_{(2)} \omega\right)^{2} \leq\left\|_{(1)} \omega\right\|_{\alpha}^{2}\left\|_{(2)} \omega\right\|_{\alpha}^{2},
$$

so we obtain

$$
I_{(1)} \omega \odot_{(2)} \omega \mid \leq\left\|_{(1)} \omega\right\|_{\alpha}\left\|_{(2)} \omega\right\|_{\alpha} .
$$

The expression (45) is called the Schwarz's $\alpha$-generalized inequality. When $b=1$ we have $\epsilon={ }_{(1)} \omega+{ }_{(2)} \omega$. By replacing $\left(_{(1)} \omega \odot_{(2)} \omega\right)$ with $\left\|_{(1)} \omega\right\|_{\alpha}\left\|_{(2)} \omega\right\|_{\alpha}$ into (44) we have the square of a binomial given by

$$
\left\|_{(1)} \omega+{ }_{(2)} \omega\right\|_{\alpha}^{2}=\left\|_{(1)} \omega\right\|_{\alpha}^{2}+2\left\|_{(1)} \omega\right\|_{\alpha}\left\|_{(2)} \omega\right\|_{\alpha}+\left\|_{(2)} \omega\right\|_{\alpha}^{2},
$$

so we obtain

$$
\left\|_{(1)} \omega+{ }_{(2)} \omega\right\|_{\alpha} \leq\left\|_{(1)} \omega\right\|_{\alpha}+\left\|_{(2)} \omega\right\|_{\alpha} .
$$

The expression (46) is called the $\alpha$-triangle inequality. Dividing by $\left\|_{(1)} \omega\right\|_{\alpha}\left\|_{(2)} \omega\right\|_{\alpha}$ both sides of (45) we have

$$
\left|\frac{{ }_{(1)} \omega \odot_{(2)} \omega}{\left\|_{(1)} \omega\right\|_{\alpha}\left\|_{(2)} \omega\right\|_{\alpha}}\right| \leq 1,
$$

that is to say,

$$
-1 \leq \frac{{ }_{(1)} \omega \odot_{(2)} \omega}{\left\|_{(1)} \omega\right\|_{\alpha}\left\|_{(2)} \omega\right\|_{\alpha}} \leq 1,
$$


so there exists a unique angle $\gamma$ such that $0 \leq \gamma \leq \pi$ and such that

$$
\cos \gamma=\frac{{ }_{(1)} \omega \odot_{(2)} \omega}{\left\|_{(1)} \omega\right\|_{\alpha}\left\|_{(2)} \omega\right\|_{\alpha}} .
$$

It is possible to define this angle to be the angle between ${ }_{(1)} \omega$ and ${ }_{(2)} \omega$. By considering the expression (42) it is also possible to define it to be the angle between ${ }_{(1)} \omega$ and ${ }_{(2)} \omega^{\prime}$. The two vectors ${ }_{(1)} \mathbf{t}$ and ${ }_{(2)} \mathbf{t}$ represent the two transformed random quantities ${ }_{1} \Omega$ and ${ }_{2} t$ defined on ${ }_{1} \Omega$ and ${ }_{2} \Omega$. Then, their $\alpha$-product is given by

$$
{ }_{(1)} \mathbf{t} \odot_{(2)} \mathbf{t}={ }_{(1)} t_{(2)}^{i_{1}} t_{i_{1}}={ }_{(1)} t^{i_{1}}{ }_{(2)} t^{i_{2}} p_{i_{2} i_{1}} .
$$

It represents the covariance of ${ }_{1} \Omega$ and ${ }_{2} \Omega$ in a vectorial fashion. When one considers the expression (47) connected with ${ }_{(1)} \mathbf{t}$ and $(2) \mathbf{t}$ it becomes

$$
\cos \gamma=\frac{{ }_{(1)} \mathbf{t} \odot{ }_{(2)} \mathbf{t}}{\left\|_{(1)} \mathbf{t}\right\|_{\alpha}\left\|_{(2)} \mathbf{t}\right\|_{\alpha}} .
$$

It expresses the Pearson $\alpha$-generalized correlation coefficient.

\section{A Metric on a Two-Dimensional Parameter Space}

We deal with a set denoted by ${ }_{(2)} S^{(2) \wedge}$ whose elements are antisymmetric tensors of order 2 . Nevertheless, by introducing the notion of $\alpha$-product of two antisymmetric tensors of order 2 we must underline a very important point: it is not necessary to refer to the bivariate random quantity $\Omega_{12}$ in order to introduce that antisymmetric tensor whose covariant components are represented like into the expression (39). Therefore, it is also possible to consider a bivariate random quantity denoted by $\Omega_{34}$ as well as an antisymmetric tensor of order 2 denoted by ${ }_{34} f$ whose covariant components are expressed by

$$
{ }_{34} f_{\left(i_{1} i_{2}\right)}=\left|\begin{array}{ll}
{ }_{(3)} \theta_{i_{1}} & { }_{(3)} \theta_{i_{2}} \\
{ }_{(4)} \theta_{i_{1}} & { }_{(4)} \theta_{i_{2}}
\end{array}\right|=\left|\begin{array}{ll}
{ }_{(3)} \theta^{i_{2}} p_{i_{2} i_{1}} & { }_{(3)} \theta^{i_{1}} p_{i_{1} i_{2}} \\
{ }_{(4)} \theta^{i_{2}} p_{i_{2} i_{1}} & { }_{(4)} \theta^{i_{1}} p_{i_{1} i_{2}}
\end{array}\right| .
$$

Thus, it is possible to extend to the antisymmetric tensors ${ }_{12} f$ and ${ }_{34} f$ the notion of $\alpha$-product. We are evidently able to point out another very important point: the domain of possible alternatives depends on information of a given individual at a certain instant even if it is always objective (de Finetti, 1989). This means that he does not express his subjective opinion in terms of probability on what is uncertain for him but he makes explicit what he knows or what he does not know at a certain instant with a given set of information. The knowledge and the ignorance of a given individual at a certain instant determine the extent of the range of the possible, so this range could also become smaller at a later time when the knowledge increases or it could otherwise become larger. With regard to the problem that we consider, there exists a more or less large number of possible alternatives. Such a number increases at a later time. If current information and knowledge of a given decision-maker do not allow him of excluding some of them as impossible, then all alternatives that can logically be considered at present remain possible for him in the sense that they are not either certainly true or certainly false. Moreover, we must suppose that $\Omega_{12}$ and $\Omega_{34}$ have at least a possible value that is the same. This common value is the true value to be verified "a posteriori". Then, we have

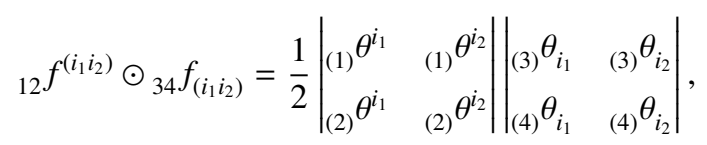

where it appears $\frac{1}{2}$ because we have always two permutations into the two determinants: one of these permutations is "good" when it turns out to be $i_{1}<i_{2}$ with respect to ${ }_{(1)} \theta_{(2)}^{i_{1}} \theta^{i_{2}}$ and ${ }_{(3)} \theta_{i_{1}(4)} \theta_{i_{2}}$, while the other is "bad" because it turns out to be $i_{2}>i_{1}$ with respect to ${ }_{(1)} \theta_{(2)}^{i_{2}} \theta^{i_{1}}$ and ${ }_{(3)} \theta_{i_{2}(4)} \theta_{i_{1}}$. Hence, we are in need of returning to normality by means of $\frac{1}{2}$. Such a normality is evidently represented by $i_{1}<i_{2}$. We can also say that it appears $\frac{1}{2 !=2}$ because we deal with antisymmetric tensors of order 2 for which it turns out to be $2 !=2$. We need different affine tensors of order 2 in order to make that calculation expressed by (51). These tensors of the joint probabilities allow us of defining the bivariate random quantities $\Omega_{13}, \Omega_{14}, \Omega_{23}$ and $\Omega_{24}$ having at least a possible value that is the same. This common value is the true value to be verified "a posteriori". Thus, we have

$$
{ }_{12} f \odot_{34} f=\left|\begin{array}{ll}
{ }_{(1)} \theta^{i_{1}}{ }_{(3)} \theta^{i_{2}} p_{i_{2} i_{1}}^{(13)} & { }_{(1)} \theta^{i_{2}}{ }_{(4)} \theta^{i_{1}} p_{i_{1} i_{2}}^{(14)} \\
{ }_{(2)} \theta^{i_{1}}{ }_{(3)} \theta^{i_{2}} p_{i_{2} i_{1}}^{(23)} & { }_{(2)} \theta^{i_{2}}{ }_{(4)} \theta^{i_{1}} p_{i_{1} i_{2}}^{(24)}
\end{array}\right| .
$$

In particular, the $\alpha$-norm of the tensor ${ }_{12} f$ is given by

$$
\left\|_{12} f\right\|_{\alpha}^{2}={ }_{12} f \odot{ }_{12} f={ }_{12} f^{\left(i_{1} i_{2}\right)}{ }_{12} f_{\left(i_{1} i_{2}\right)},
$$


so it turns out to be

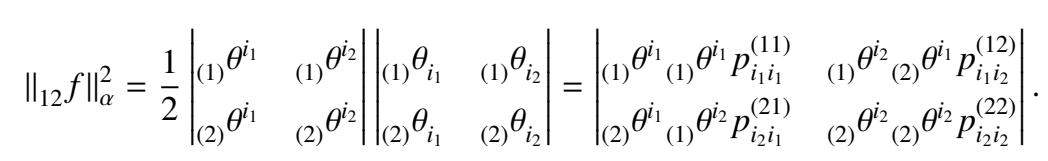

Anyway, it is always possible to write

$$
{ }_{12} f \odot_{34} f=\left|\begin{array}{ll}
{ }_{11} \omega \odot_{(3)} \omega & { }_{(1)} \omega \odot_{(4)} \omega \\
{ }_{(2)} \omega \odot_{(3)} \omega & { }_{(2)} \omega \odot_{(4)} \omega
\end{array}\right|
$$

as well as

$$
\left\|_{12} f\right\|_{\alpha}^{2}=\left|\begin{array}{cc}
\left\|_{(1)} \omega\right\|_{\alpha}^{2} & { }_{(1)} \omega \odot_{(2)} \omega \\
\odot_{(2)} \omega \odot_{(1)} \omega & \left\|_{(2)} \omega\right\|_{\alpha}^{2}
\end{array}\right| .
$$

The $\alpha$-norm of the tensor ${ }_{12} f$ is again strictly positive. It is equal to 0 when the components of ${ }_{12} f$ are null. Nevertheless, this does not mean that the components of the two vectors founding the tensor are null. Indeed, it suffices that one writes ${ }_{(1)} \omega=b_{(2)} \omega$, with $b \in \mathbb{R}$, in order to obtain

$$
\left\|_{12} f_{b}\right\|_{\alpha}^{2}=\frac{1}{2}\left|\begin{array}{cc}
b_{(2)} \theta^{i_{1}} & b_{(2)} \theta^{i_{2}} \\
{ }_{(2)} \theta^{i_{1}} & { }_{(2)} \theta^{i_{2}}
\end{array}\right|\left|\begin{array}{cc}
b_{(2)} \theta_{i_{1}} & b_{(2)} \theta_{i_{2}} \\
{ }_{(2)} \theta_{i_{1}} & { }_{(2)} \theta_{i_{2}}
\end{array}\right|=\left|\begin{array}{cc}
b^{2}\left\|_{(2)} \omega\right\|_{\alpha}^{2} & b\left\|_{(2)} \omega\right\|_{\alpha}^{2} \\
b\left\|_{(2)} \omega\right\|_{\alpha}^{2} & \left\|_{(2)} \omega\right\|_{\alpha}^{2}
\end{array}\right|=0 .
$$

The $\alpha$-norm of the tensor ${ }_{12} f$ evidently implies that $\Omega_{12}$ and $\Omega_{12}$ have all possible values that are the same. One and only one of these possible values will be the true value to be verified "a posteriori". We define a tensor $f$ as a linear combination of ${ }_{12} f$ and ${ }_{34} f$ such that we can write $f={ }_{12} f+b_{34} f$, with $b \in \mathbb{R}$. Then, the Schwarz's $\alpha$-generalized inequality becomes

$$
\left.\right|_{12} f \odot_{34} f \mid \leq\left\|_{12} f\right\|_{\alpha}\left\|_{34} f\right\|_{\alpha},
$$

the $\alpha$-triangle inequality becomes

$$
\left\|_{12} f+{ }_{34} f\right\|_{\alpha} \leq\left\|_{12} f\right\|_{\alpha}+\left\|_{34} f\right\|_{\alpha},
$$

while the cosine of the angle $\gamma$ becomes

$$
\cos \gamma=\frac{{ }_{12} f \odot_{34} f}{\left\|_{12} f\right\|_{\alpha}\left\|_{34} f\right\|_{\alpha}} .
$$

It is possible to consider two univariate transformed random quantities that are respectively ${ }_{1} t_{\Omega}$ and ${ }_{2} t$. They are represented by ${ }_{(1)} \mathbf{t}$ and ${ }_{(2)} \mathbf{t}$ whose contravariant components are given by ${ }_{(1)} t^{i}={ }_{(1)} \theta^{i}-{ }_{(1)} \bar{\omega}^{i}$ and ${ }_{(2)} t^{i}={ }_{(2)} \theta^{i}-{ }_{(2)} \bar{\omega}^{i}$. Therefore, it is possible to introduce an antisymmetric tensor of order 2 denoted by ${ }_{12} t$ characterizing a bivariate transformed random quantity denoted by $\Omega_{\Omega_{12}} t$. Then, the contravariant components of this tensor are given by

$$
12^{t^{\left(i_{1} i_{2}\right)}}=\left|\begin{array}{ll}
(1)^{i_{1}} & (1)^{t_{2}} \\
(2)^{i_{1}} & (2)^{t_{2}}
\end{array}\right| .
$$

Its covariant components are given by

$$
{ }_{12} t_{\left(i_{1} i_{2}\right)}=\left|\begin{array}{ll}
{ }_{(1)} t_{i_{1}} & { }_{(1)} t_{i_{2}} \\
{ }_{(2)} t^{t_{1}} & { }_{(2)} t_{i_{2}}
\end{array}\right|=\left|\begin{array}{ll}
{ }_{(1)} t^{i_{2}} p_{i_{2} i_{1}} & { }_{(1)} t^{i_{1}} p_{i_{1} i_{2}} \\
{ }_{(2)} t^{i_{2}} p_{i_{2} i_{1}} & { }_{(2)} t^{i_{1}} p_{i_{1} i_{2}}
\end{array}\right| .
$$

The $\alpha$-product of the two tensors ${ }_{12} t$ and ${ }_{34} t$ is given by

$$
{ }_{12} t \odot_{34} t=\left|\begin{array}{ll}
{ }_{(1)} \mathbf{t} \odot_{(3)} \mathbf{t} & { }_{(1)} \mathbf{t} \odot_{(4)} \mathbf{t} \\
{ }_{(2)} \mathbf{t} \odot_{(3)} \mathbf{t} & { }_{(2)} \mathbf{t} \odot_{(4)} \mathbf{t}
\end{array}\right| .
$$

The $\alpha$-norm of the tensor ${ }_{12} t$ is given by

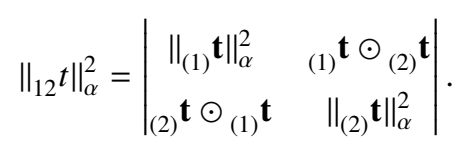


The cosine of the angle $\gamma$ is given by

$$
\cos \gamma=\frac{{ }_{12} t \odot_{34} t}{\left\|_{12} t\right\|_{\alpha}\left\|_{34} t\right\|_{\alpha}} .
$$

All these expressions are useful in order to characterize meaningful quantitative relationships between multiple random quantities. We always need them when we study a parameter space whose unknown parameter is not a real number but it is an $m$-tuple of real numbers, with $m \geq 2$.

\section{Metric Properties of the Expected Value Into a Two-Dimensional Parameter Space}

The criterion of the expected value considered into decision theory under conditions of uncertainty is connected with a loss function. It always depends on a parameter belonging to a parameter space denoted by $\Omega$. Such a summarizing criterion evidently coincides with the notion of prevision of a bivariate random quantity when we deal with a two-dimensional parameter space. Hence, we study metric properties of the expected value into a two-dimensional parameter space. The notion of $\alpha$-product depends on three elements that are two vectors of $E_{m},_{(1)} \omega$ and ${ }_{(2)} \omega$, and one affine tensor $p=\left(p_{i_{1} i_{2}}\right)$ of order 2 belonging to $E_{m}^{(2)}=E_{m} \otimes E_{m}$. Given any ordered pair of vectors, $p$ is uniquely determined as a geometric object. This implies that each covariant component of $p$ is always a subjective probability intrinsically coherent (de Finetti, 1980). It is possible that all reasonable people share each covariant component of $p$ with regard to some problem of decision theory that may be considered. Nevertheless, an opinion in terms of probability shared by many people always remains a subjective opinion. It is meaningless to say that it is objectively exact. Indeed, a sum of many subjective opinions in terms of probability can never lead to an objectively correct conclusion (de Finetti, 1972). Thus, given a bivariate random quantity $\left.\Omega_{12} \equiv{ }_{1} \Omega,{ }_{2} \Omega\right\}$, its coherent prevision $\mathbf{P}\left(\Omega_{12}\right)$ is an $\alpha$-product whose metric properties remain unchanged by extending them to $\mathbf{P}$. Therefore, $\mathbf{P}$ is an $\alpha$-commutative prevision because it is possible to write

$$
\mathbf{P}\left({ }_{1} \Omega_{2} \Omega\right)=\mathbf{P}\left({ }_{2} \Omega{ }_{1} \Omega\right),
$$

$\mathbf{P}$ is an $\alpha$-associative prevision because it is possible to write

$$
\mathbf{P}\left[\left(b_{1} \Omega\right)_{2} \Omega\right]=\mathbf{P}\left[{ }_{1} \Omega\left(b_{2} \Omega\right)\right]=b \mathbf{P}\left({ }_{1} \Omega_{2} \Omega\right), \forall b \in \mathbb{R},
$$

$\mathbf{P}$ is an $\alpha$-distributive prevision because it is possible to write

$$
\mathbf{P}\left[\left({ }_{1} \Omega+{ }_{2} \Omega\right)_{3} \Omega\right]=\mathbf{P}\left({ }_{1} \Omega_{3} \Omega\right)+\mathbf{P}\left({ }_{2} \Omega_{3} \Omega\right) .
$$

Moreover, when one writes

$$
\mathbf{P}\left({ }_{1} \Omega_{2} \Omega\right)=\mathbf{P}\left({ }_{2} \Omega_{1} \Omega\right)=0,
$$

one says that ${ }_{1} \Omega$ and ${ }_{2} \Omega$ are $\alpha$-orthogonal univariate random quantities. We exclude that all possible values of $\Omega$ and ${ }_{2} \Omega$ are null. In particular, one observes that the $\alpha$-distributive property of prevision implies that the covariant components of the affine tensor $p^{(13)}$ are equal to the ones of the affine tensor $p^{(23)}$. Moreover, the covariant components of the affine tensor connected with the two univariate random quantities ${ }_{1} \Omega+{ }_{2} \Omega$ and ${ }_{3} \Omega$ are the same of the ones of $p^{(13)}$ and $p^{(23)}$. By considering a bivariate random quantity one finally says that its prevision $\mathbf{P}$ is bilinear. If the possible values of the two univariate random quantities of $\left.\Omega_{12} \equiv{ }_{1} \Omega,{ }_{2} \Omega\right\}$ are correspondingly equal and the covariant components of the tensor $p=\left(p_{i_{1} i_{2}}\right)$ having different numerical values as indices are null, then $\mathbf{P}\left(\Omega_{12}\right)=\mathbf{P}\left({ }_{1} \Omega{ }_{2} \Omega\right)=\mathbf{P}\left({ }_{2} \Omega{ }_{1} \Omega\right)$ coincides with the $\alpha$-norm of ${ }_{(1)} \omega={ }_{(2)} \omega$. When $\mathbf{P}\left(\Omega_{12}\right)$ is a coherent prevision of $\Omega_{12} \equiv\left\{_{1} \Omega,{ }_{2} \Omega\right\}$ it follows that its marginal univariate random quantities, ${ }_{1} \Omega$ and ${ }_{2} \Omega$, represent two separate and finite partitions of incompatible and exhaustive events whose non-negative probabilities sum to 1 . Thus, conditions of coherence coincide with non-negativity and finite additivity (de Finetti, 1975), (Coletti, Scozzafava \& Vantaggi, 2015), (Coletti, Petturiti \& Vantaggi, 2014). We note a very important point: metric properties of the expected value allow us of rewriting some fundamental metric expressions. Thus, when we rewrite (55) and (56) it turns out to be

$$
{ }_{12} f \odot_{34} f=\left|\begin{array}{ll}
\mathbf{P}\left({ }_{1} \Omega_{3} \Omega\right) & \mathbf{P}\left({ }_{1} \Omega_{4} \Omega\right) \\
\mathbf{P}\left({ }_{2} \Omega_{3} \Omega\right) & \mathbf{P}\left({ }_{2} \Omega_{4} \Omega\right)
\end{array}\right|
$$

and

$$
\left\|_{12} f\right\|_{\alpha}^{2}=\left|\begin{array}{ll}
\mathbf{P}\left({ }_{1} \Omega_{1} \Omega\right) & \mathbf{P}\left({ }_{1} \Omega_{2} \Omega\right) \\
\mathbf{P}\left({ }_{2} \Omega_{1} \Omega\right) & \mathbf{P}\left({ }_{2} \Omega_{2} \Omega\right)
\end{array}\right| .
$$

Thus, it is evidently possible to realize that fundamental metric expressions involving antisymmetric tensors of order 2 are based on the notion of coherent prevision of a bivariate random quantity. This means that we are able to divide a given

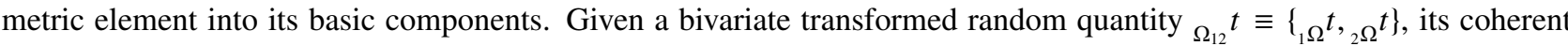


prevision $\mathbf{P}\left(\Omega_{\Omega_{12}} t\right)$ is again an $\alpha$-product whose metric properties remain unchanged by extending them to $\mathbf{P}$. In particular, when it turns out to be $p_{i_{1} i_{2}}=p_{i_{1}} p_{i_{2}}, \forall i_{1}, i_{2} \in I_{m}$, with $I_{m} \equiv\{1,2, \ldots, m\}$, one observes that a stochastic independence exists. Hence, one obtains $\mathbf{P}\left(\Omega_{\Omega_{12}} t\right)=0$, that is to say, (1) $\mathbf{t}$ and ${ }_{(2)} \mathbf{t}$ are $\alpha$-orthogonal. One equivalently says that the covariance of ${ }_{1} \Omega$ and ${ }_{2} \Omega$ is equal to 0 .

\section{The Bayes' Theorem Connected With a Two-Dimensional Parameter Space}

Given a bivariate random quantity denoted by $\left.\Omega_{12} \equiv{ }_{1} \Omega,{ }_{2} \Omega\right\}$, its univariate elements are ${ }_{1} \Omega$ and ${ }_{2} \Omega$. Each covariant component of $p=\left(p_{i_{1} i_{2}}\right)$ represents a probability of the joint of two events. This probability includes a conditional probability of an event given the other. Hence, by denoting by $E_{i}, i=1, \ldots, m$, the possible values of ${ }_{1} \Omega$ and by $C_{j}$, $j=1, \ldots, m$, the possible values of ${ }_{2} \Omega$, where the possible values of the two univariate random quantities are expressed by means of the same scale of measurement, it turns out to be, for instance, $\mathbf{P}\left(E_{1} \wedge C_{1}\right)=\mathbf{P}\left(C_{1}\right) \mathbf{P}\left(E_{1} \mid C_{1}\right)$, where $E_{1} \wedge C_{1}$ is a logical product of two events and $\mathbf{P}\left(E_{1} \wedge C_{1}\right)$ is a probability of their joint. The same thing evidently goes with respect to each covariant component of $p$ (Coletti, Petturiti \& Vantaggi, 2016a), (Coletti, Petturiti \& Vantaggi, 2016b), (Gilio \& Sanfilippo, 2014). We note that $\left(E_{1} \mid C_{1}\right)$ is a tri-event whose values are $1|1=1,0| 1=0,0|0=1| 0=\emptyset$ according to the logic of certainty. Any tri-event can always be expressed by means of two events. Thus, any tri-event represents only a formal variation with respect to the starting delimitation because $\emptyset=$ void is added to the two starting values $1=$ true and $0=$ false. From the notion of conditional probability it follows that the notion of probability is relative to the current state of information of a given decision-maker. Thus, by considering $\mathbf{P}\left(E_{1} \mid C_{1}\right)$, the current state of information of a given decision-maker is represented by $C_{1}$. Any bivariate random quantity representing a two-dimensional parameter space allows us of partitioning a random element coinciding with one of the two unknown parameters under consideration. Therefore, we consider the possible values of $\Omega$ as a partition of this random element. A prior probability law coincides with vector homographies obtained by means of the covariant components of $p$ that we can consider into a two-way table. We denote this probability law by $\mathbf{P}\left(C_{j}\right), j=1, \ldots, m$, where $\mathbf{P}\left(C_{1}\right), \ldots, \mathbf{P}\left(C_{m}\right)$ are marginal probabilities connected with the possible values of ${ }_{2} \Omega$. We can know $\mathbf{P}\left(E_{i} \mid C_{j}\right), i=j=1, \ldots, m$, because we obtain

$$
\mathbf{P}\left(E_{i} \mid C_{j}\right)=\frac{\mathbf{P}\left(E_{i} \wedge C_{j}\right)}{\mathbf{P}\left(C_{j}\right)}
$$

by using the covariant components of $p$ contained into the two-way table under consideration. Now, we can obtain a posterior probability law by applying the Bayes' theorem. Thus, we have

$$
\mathbf{P}\left(C_{j} \mid E_{i}\right)=\frac{\mathbf{P}\left(C_{j} \wedge E_{i}\right)}{\mathbf{P}\left(E_{1} \wedge C_{1}\right)+\ldots+\mathbf{P}\left(E_{m} \wedge C_{m}\right)},
$$

where it turns out to be $i=j=1, \ldots, m$. It is essential to note that we can observe a posterior probability law because we use a geometric approach that allows us of dividing a given random element into its essential and simpler components. The number of these components is equal to $m$.

\section{Conclusions}

We have proposed an original mathematical model according to a Bayesian approach to decision theory explaining uncertainty from a point of view connected with vector spaces. An one-dimensional parameter space has been represented by means of a univariate random quantity. A two-dimensional parameter space has been represented by means of a bivariate random quantity. We have supposed that every univariate random quantity is bounded from above and below. This thing is not a loss of generality. Indeed, a given individual may always establish which is the lowest possible value of a parameter space as well as the greatest possible value of it at a certain instant with a given set of information. We have accepted the principles of the theory of concordance into the domain of subjective probability. This thing has never been made before. We have considered the range of possibility of a given individual at a certain instant with a given set of information. Such a range is not a $\sigma$-algebra. We have consequently distributed probabilities as masses over it. Thus, a parameter space has been provided with a geometric structure. We have introduced coherent probability laws characterizing random quantities after considering ranges of possibility. These probability laws can always be summarized by means of the notion of prevision of a univariate or bivariate random quantity. We have observed that metric properties of the notion of $\alpha$-product mathematically fulfill the ones of a coherent prevision of a bivariate random quantity. We have shown fundamental metric expressions connected with transformed random quantities representing changes of origin. We have obtained a posterior probability law by applying the Bayes' theorem into a geometric context connected with a two-dimensional parameter space.

\section{References}

Barlow, R. E., Claroti, C. A., \& Spizzichino, F. (1993). Reliability and decision making. Boca Raton: CRC Press.

Basu, D. (1975). Statistical information and likelihood. Sankhya A, 37, 1-71. 
Castellano, V. (1989). Sociological works. Roma: Università degli studi di Roma "La Sapienza".

Coletti, G., \& Scozzafava, R. (2002). Probabilistic logic in a coherent setting. Dordrecht/Boston/London: Kluwer Academic Publishers.

Coletti, G., Petturiti, D., \& Vantaggi, B. (2016a). When upper conditional probabilities are conditional possibility measures. Fuzzy Sets and Systems, 304, 45-64.

Coletti, G., Petturiti, D., \& Vantaggi, B. (2016b). Conditional belief functions as lower envelopes of conditional probabilities in a finite setting. Information Sciences, 339, 64-84.

Coletti, G., Scozzafava, R., \& Vantaggi, B. (2015). Possibilistic and probabilistic logic under coherence: default reasoning and System P. Mathematica Slovaca, 65(4), 863-890.

Coletti, G., Petturiti, D., \& Vantaggi, B. (2014). Bayesian inference: the role of coherence to deal with a prior belief function. Statistical Methods $\mathcal{E}$ Applications, 23(4), 519-545.

de Finetti, B. (1975). Theory of probability, 2. London-New York-Sydney-Toronto: J. Wiley \& Sons.

de Finetti, B. (1972). Probability, Induction and Statistics (The art of guessing). London-New York-Sydney-Toronto: J. Wiley \& Sons.

de Finetti, B. (1980). Probability: beware of falsifications!. In H. E. Kyburg, jr., \& H. E. Smokler. Studies in subjective probability, 195-224. Huntington, New York: R. E. Krieger Publishing Company.

de Finetti, B. (1981). The role of "Dutch Books" and of "proper scoring rules". The British Journal of Psychology of Sciences, 32, 55-56. https:// doi.org/10.1093/bjps/32.1.55

de Finetti, B. (1982a). Probability: the different views and terminologies in a critical analysis. Logic, Methodology and Philosophy of Science VI (Hannover, 1979), 391-394.

de Finetti, B. (1982b). The proper approach to probability. In G. Koch, \& F. Spizzichino. Exchangeability in Probability and Statistics, 1-6. Amsterdam: North-Holland Publishing Company.

de Finetti, B. (1989). Probabilism: A Critical Essay on the Theory of Probability and on the Value of Science. Erkenntnis, $31(2 / 3), 169-223$.

de Finetti, B. (2011). Probability and statistics in relation to induction, from various points of view. In B. de Finetti (Ed.) Induction and statistics (C. I. M. E. Summer Schools, 18), 1-122. Heidelberg: Springer.

deGroot, M. H. (1962). Uncertainty, information and sequential experiments. The Annals of Mathematical Statistics, 33, 404-419.

Forcina, A. (1982). Gini's contributions to the theory of inference. International Statistical Review, 50, 65-70. https://doi.org/10.2307/1402459

Gili, A., \& Bettuzzi, G. (1986). About concordance square indexes among deviations: correlation indexes. Statistica, 46(1), 17-46.

Gilio, A., \& Sanfilippo, G. (2014). Conditional random quantities and compounds of conditionals. Studia logica, 102(4), 709-729.

Gini, C. (1966). Statistical methods. Roma: Istituto di statistica e ricerca sociale "Corrado Gini". Università degli studi di Roma.

Gini, C. (1926). The contributions of Italy to modern statistical methods. Journal of the Royal Statistical Society, 89(4), 703-724.

Good, I. J. (1962). Subjective probability as the measure of a non-measureable set. In E. Nagel, P. Suppes, \& A. Tarski. Logic, Methodology and Philosophy of Science, 319-329. Stanford: Stanford University Press.

Jeffreys, H. (1961). Theory of probability, 3rd edn.. Oxford: Clarendon Press.

Koopman, B. O. (1940). The axioms and algebra of intuitive probability. Annals of Mathematics, 41, 269-292. https: // doi.org/10.2307/1969003

Kyburg jr., H. E., \& Smokler, H. E. (1964). Studies in subjective probability. New York, London, Sydney: J. Wiley \& Sons.

McCullagh, P. (1987). Tensor methods in statistics. London-New York: Chapman and Hall. 
Piccinato, L. (1986). de Finetti's logic of uncertainty and its impact on statistical thinking and practice, a cura di P. K.

Goel e A. Zellner, North-Holland, Amsterdam. Bayesian Inference and Decision Techniques, 13-30.

Pistone, G., Riccomagno, E., \& Wynn, H. P. (2001). Algebraic statistics. Boca Raton-London-New York-Washington, D.C.: Chapman \& Hall.

Pompilj, G. (1957). On intrinsic independence. Bulletin of the International Statistical Institute, 35(2), 91-97.

Ramsey, F. P. (1960). The foundations of mathematics and other logical essays. Paterson, N. J.: Littlefield, Adams \& Co. Savage, L. J. (1954). The foundations of statistics. New York: J. Wiley \& Sons.

\section{Copyrights}

Copyright for this article is retained by the author(s), with first publication rights granted to the journal.

This is an open-access article distributed under the terms and conditions of the Creative Commons Attribution license (http://creativecommons.org/licenses/by/4.0/). 